Review article

\title{
Identification and diagnosis of patients with familial chylomicronaemia syndrome (FCS): Expert panel recommendations and proposal of an "FCS score"
}

Philippe Moulin a ${ }^{a}$, Robert Dufour ${ }^{b}$, Maurizio Averna ${ }^{c}$, Marcello Arca ${ }^{d}$, Angelo B Cefalì c Davide Noto ${ }^{c}$, Laura D'Erasmo ${ }^{\mathrm{d}}$, Alessia Di Costanzo ${ }^{\mathrm{d}}$, Christophe Marçais ${ }^{\mathrm{a}}$, Luis Antonio Alvarez-Sala Walther ${ }^{\mathrm{e}}$, Maciej Banach ${ }^{\mathrm{f}}$, Jan Borén ${ }^{\mathrm{g}}$, Robert Cramb ${ }^{\mathrm{h}}$, Ioanna Gouni-Berthold ${ }^{\mathrm{i}}$, Elizabeth Hughes ${ }^{\mathrm{j}}$, Colin Johnson ${ }^{\mathrm{k}}$, Xavier Pintó ${ }^{ }$, Željko Reiner ${ }^{\mathrm{m}}$, Jeanine Roeters van Lennep ${ }^{\mathrm{n}}$, Handrean Soran ${ }^{\mathrm{o}}$, Claudia Stefanutti ${ }^{\mathrm{p}}$, Erik Stroes ${ }^{\mathrm{q}}$, Eric Bruckert ${ }^{\mathrm{r}, *}$

\footnotetext{
${ }^{a}$ Hôpital Cardiovasculaire Louis Pradel, Hospices Civils de Lyon, INSERM UMR 1060 Carmen, Université Claude Bernard Lyon 1, Lyon, France

b Institut de Recherches Cliniques de Montréal, Montréal, Canada

${ }^{c}$ Dipartimento Biomedico di Medicina Interna e Specialistica (Di.Bi.M.I.S.), University of Palermo, Palermo, Italy

${ }^{\mathrm{d}}$ Department of Internal Medicine and Medical Specialties, Sapienza University of Rome, Rome, Italy

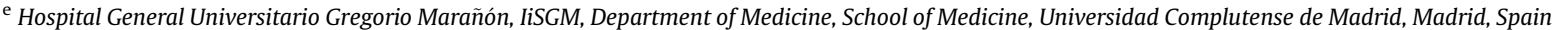

${ }^{\mathrm{f}}$ Medical University of Lodz, Lodz, Poland

${ }^{g}$ University of Gothenburg, Gothenburg, Sweden

${ }^{\mathrm{h}}$ University Hospitals Birmingham NHS Foundation Trust, Birmingham, UK

i Polyclinic for Endocrinology, Diabetes, and Preventive Medicine, University of Cologne, Cologne, Germany

${ }^{j}$ Sandwell and West Birmingham Hospitals NHS Trust, Birmingham, UK

${ }^{\mathrm{k}}$ University Hospital, Southampton, UK

${ }^{1}$ Bellvitge University Hospital, Barcelona, Spain

${ }^{\mathrm{m}}$ University Hospital Center Zagreb, School of Medicine, University of Zagreb, Zagreb, Croatia

${ }^{\mathrm{n}}$ Erasmus Medical Centre, Rotterdam, the Netherlands

- Central Manchester University Hospital NHS Foundation Trust, Manchester, UK

${ }^{\mathrm{p}}$ Department of Molecular Medicine, Sapienza University of Rome, Rome, Italy

${ }^{\mathrm{q}}$ Academic Medical Center, Amsterdam, the Netherlands

${ }^{\mathrm{r}}$ Hôpital Pitié Salpêtrière, Paris, France
}

\section{A R T I C L E I N F O}

\section{Article history:}

Received 29 March 2018

Received in revised form 23 May 2018

Accepted 13 June 2018

Available online 18 June 2018

\section{Keywords:}

Familial chylomicronaemia syndrome

Major hypertriglyceridaemia

Multifactorial chylomicronaemia

Acute pancreatitis

\begin{abstract}
A B S T R A C T
Familial chylomicronaemia syndrome (FCS) is a rare, inherited disorder characterised by impaired clearance of triglyceride (TG)-rich lipoproteins from plasma, leading to severe hypertriglyceridaemia (HTG) and a markedly increased risk of acute pancreatitis. It is due to the lack of lipoprotein lipase (LPL) function, resulting from recessive loss of function mutations in the genes coding LPL or its modulators. A large overlap in the phenotype between FCS and multifactorial chylomicronaemia syndrome (MCS) contributes to the inconsistency in how patients are diagnosed and managed worldwide, whereas the incidence of acute hypertriglyceridaemic pancreatitis is more frequent in FCS. A panel of European experts provided guidance on the diagnostic strategy surrounding FCS and proposed an algorithm-based diagnosis tool for identification of these patients, which can be readily translated into practice. Features included in this FCS score comprise: severe elevation of plasma TGs (fasting TG levels $>10 \mathrm{mmol} / \mathrm{L}$ [885 mg/dL] on multiple occasions), refractory to standard TG-lowering therapies, a young age at onset, the lack of secondary factors (except for pregnancy and oral oestrogens) and a history of episodes of acute pancreatitis. Considering 53 FCS patients from three cohorts and 52 MCS patients from three cohorts, the overall sensitivity of the FCS score $(\geq 10)$ was $88 \%$ (95\% confidence interval [CI]: $0.76,0.97$ ) with an overall specificity of $85 \%(95 \% \mathrm{CI}$ : $0.75,0.94)$. Receiver operating characteristic curve area was
\end{abstract}

\footnotetext{
* Corresponding author. Endocrinologie Métabolisme et Prévention Cardiovasculaire, Institut E3M et IHU Cardiométabolique (ICAN), Hôpital Pitié Salpêtrière, 47-83 Boulevard de l'Hôpital, 75013, Paris, France.

E-mail address: eric.bruckert@aphp.fr (E. Bruckert).
} 
0.91. Pragmatic clinical scoring, by standardising diagnosis, may help differentiate FCS from MCS, may alleviate the need for systematic genotyping in patients with severe HTG and may help identify highpriority candidates for genotyping.

(c) 2018 The Authors. Published by Elsevier B.V. This is an open access article under the CC BY-NC-ND license (http://creativecommons.org/licenses/by-nc-nd/4.0/).

\section{Introduction}

1.1. Key issues in the identification and diagnosis of familial chylomicronaemia syndrome

Familial chylomicronaemia syndrome (FCS) is a rare, inherited disorder of triglyceride (TG)-rich lipoprotein metabolism [1]. The estimated worldwide prevalence is approximately one individual per million [2]. It often presents in childhood and has been described in all ethnicities, although a higher prevalence has been observed in some geographical areas such as Quebec, due to a founder effect [3].

Chylomicronaemia is the accumulation in the bloodstream of chylomicrons, i.e., large, TG-rich lipoprotein particles produced by enterocytes after a meal [1,2]. Under physiological conditions, chylomicrons are rapidly cleared from plasma by the action of lipoprotein lipase (LPL), an enzyme located on the endothelial surface of adipose and muscle tissues, which hydrolyses TG into fatty acids and glycerol. Chylomicrons are converted into chyloremnants, which are in turn taken up by specific cellular receptors [4]. In FCS, a lack of LPL functionality, mostly due to pathogenic variants in the set of genes involved in LPL function, markedly impairs clearance of chylomicrons from the plasma. The initial reports of FCS involved homozygous or compound heterozygous carriers of loss of function mutations in LPL and apolipoprotein C2 (APOC2) genes. Subsequently, combined loss of function mutations in genes coding for apolipoprotein A5 (APOA5), lipase maturation factor 1 (LMF1), glycosylphosphatidylinositol-anchored high-density lipoprotein-binding protein 1 (GPIHBP1) and glycerol3-phosphate dehydrogenase 1 (G3PDH1) were also identified as causal in FCS [1,5-11]. Additional genes are likely to be identified in the future, since in a large proportion of patients (more than onethird) with a phenotype similar to FCS, no deleterious variants in the aforementioned genes can be identified [12,13].

Although environmental factors increase the magnitude of hyperchylomicronaemia, they are not required for the expression of FCS phenotype; however, many cases of FCS syndrome in women are discovered at the third trimester of pregnancy. It has been consistently reported that FCS strongly increases the risk of acute pancreatitis (AP) [14]. However, it remains an elusive goal to identify patients with FCS who will develop AP versus those who will not.

A key concern is that a clear diagnosis might be missed in patients with FCS. Indeed, the condition is very rare and high levels of TGs are more often due to multifactorial chylomicronaemia syndrome (MCS) [8,15]. This syndrome results from the combination, in various proportions, of predisposing variants ranging from loss of function heterozygous mutations to likely pathogenic variants in candidate genes with co-morbidities known to raise plasma TGs (uncontrolled diabetes, hypothyroidism, pregnancy) and environmental factors (such as alcohol abuse, unhealthy diet) and certain medications (such as glucocorticoids, ethinylestradiol, neuroleptics). MCS is also characterised by an increased risk of AP but the estimated odds ratio of 50 is clearly less than the AP odds ratio of 360 reported in patients with FCS (Fig. 1) [16,17].

The apparently similar phenotype of MCS means that FCS may be missed or undiagnosed. Many patients with FCS suffer recurrent episodes of AP before the diagnosis is made. Lack of disease awareness on the part of physicians in emergency medicine, primary care and gastroenterology is a key barrier to FCS diagnosis, leading to a low rate of appropriate referrals and follow-up.

A panel of European clinical experts experienced in diagnosing FCS convened in Amsterdam in July 2017 to discuss the definition and terminology of FCS and optimal strategies for the identification, diagnosis and evaluation of patients, which could be readily translated into practice to improve the approach used to identify and diagnose FCS, which we present below.

\section{Challenges in defining FCS}

- FCS is characterised by very high plasma TG concentrations ( $>10 \mathrm{mmol} / \mathrm{L}$ or $885 \mathrm{mg} / \mathrm{dL}$ in the untreated state). A key characteristic is milky looking plasma, whereby a white chylomicron layer floats above the lactescent plasma compartment following decantation and/or low-speed centrifugation of the plasma sample. Although controversial [2], the expert panel considered that most adult patients with FCS present with both increased chylomicrons and very low-density lipoproteins (VLDLs), and, consequently, more often display type $\mathrm{V}$ than type I dyslipidaemia $[9,18,19]$. Therefore, type I phenotype has been considered to be poorly sensitive but a highly specific feature of FCS in adults. Type I phenotype is more frequently found in children with FCS, which may reflect the ability of hepatic lipase to efficiently hydrolyse VLDL-TG in children and teenagers [20]. It is noteworthy that few patients with auto-immune hyperchylomicronaemia also present with type I phenotype [5,21].

- Patients with FCS may experience physical complications including abdominal pain, which can range in intensity from mild to incapacitating [1]. Moreover, they have a high risk of developing severe recurrent AP [22,23], a potentially lifethreatening complication [24], resulting in increased morbidity and mortality [2,25], and may lead to chronic pancreatitis, permanent damage to pancreatic tissue, pancreatic insufficiency and type 3 diabetes [26,27]. Studies have highlighted a significant relationship between TG level and pancreatitis, with a $4 \%$ increased risk of AP for every $100 \mathrm{mg} / \mathrm{dL}$ or $\sim 1.1 \mathrm{mmol} / \mathrm{L}$ increase in TG level $[28,29]$. In a prospective cohort study, even mild hypertriglyceridaemia (HTG) (non-fasting TG $>177 \mathrm{mg} / \mathrm{dL}$ [2 $\mathrm{mmol} / \mathrm{L}]$ ) was associated with a graded increased risk of AP [30].

- Other clinical symptoms include transient eruptive xanthomas, which affect $<50 \%$ of individuals with FCS, often appearing on the trunk and extremities, and lipemia retinalis, a milky appearance of the retinal vessels [1,29]. Hepatosplenomegaly may also result from TG uptake by macrophages [1]. Neurological symptoms, such as irritability, memory problems, dementia and depression, have also been documented [24]. Patients with FCS tend to have a lower body weight because of restriction of their food intake owing to abdominal pain. The impact of the disease is such that quality of life and daily activities including work may be adversely affected [31,32]. 


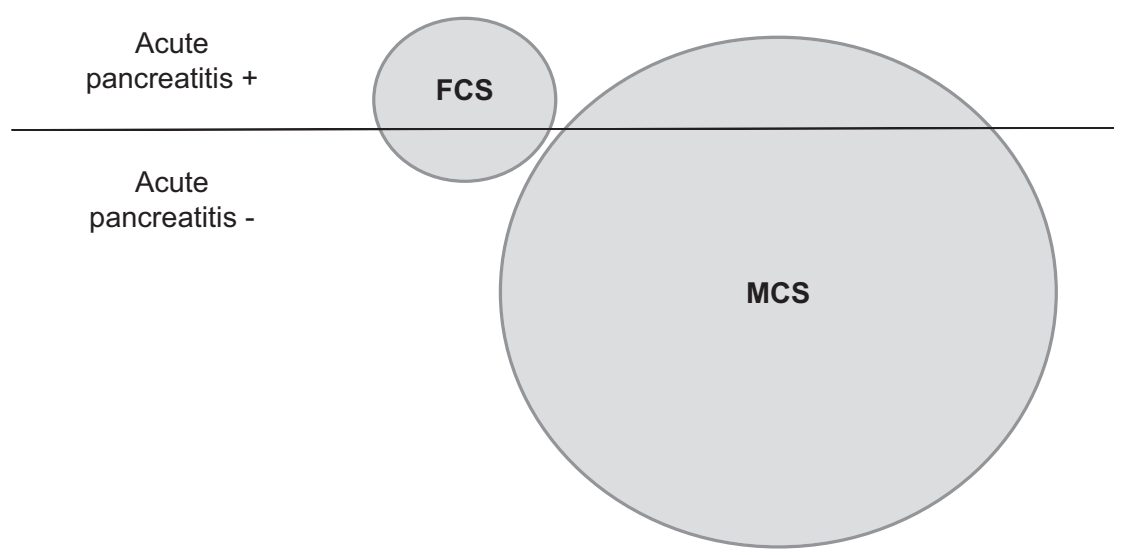

Fig. 1. Relative prevalence of pancreatitis in FCS and MCS.

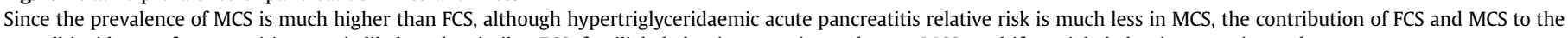
overall incidence of pancreatitis cases is likely to be similar. FCS, familial chylomicronaemia syndrome; MCS, multifactorial chylomicronaemia syndrome.

We discussed the importance of using consistent, clear and well-defined terminology when describing the condition, ensuring that this is something that will be easily identified by patients, clinical chemists and primary care physicians, in addition to specialists more familiar with the area. Of the numerous terms that have been used historically in the literature, in the clinical setting and by patient societies, use of "FCS" seems to be a pragmatic choice. Indeed, there are key patient organisations that are using this terminology, including the LPLD Alliance (http://www. lpldalliance.org/) in the UK and The FCS Foundation (https:// www.livingwithfcs.org/) in the USA. The condition should be characterised as a 'syndrome' due to the variety of symptoms present, with different presentations between individuals. The main limitation is that most of the cases are apparently sporadic, within a nuclear family, due to the recessive character of the disease. However, the preferred terminology is "familial chylomicronaemia syndrome" - abbreviated as "FCS".

\section{Diagnosis}

\subsection{Disease classification}

One of the main difficulties that we considered is the uncertain boundary between FCS and MCS, since the genetic load increases progressively from MCS, where it can be minimal, to FCS, where it is maximal in carriers of homozygous nonsense mutations in the LPL gene. The full spectrum is described in Fig. 2. We propose that FCS corresponds to a monogenic disorder with a genetic load high enough to generate constant HTG, mostly $>10 \mathrm{mmol} / \mathrm{L}(885 \mathrm{mg} / \mathrm{dL}$ ) with recurrent decompensations in the absence of obvious secondary factors (and decreased post-heparin LPL activity, although infrequently assayed in clinical practice). In addition to homozygous LPL deficiency, homozygous loss of function mutations in APOC2, APOA5, GPIHBP1, LMF1 and G3PDH1 genes should be considered, as well as compound heterozygous mutations involving $L P L$ and these genes.

Patients with MCS may have a combination of a heterozygous loss of function mutation and/or likely pathogenic frequent variants in TG-raising genes, thus producing (oligogenic/polygenic) severe HTG [8,9]. However, in such cases, hyperchylomicronaemia is often transient and low LPL activity is inconstant. Between recurrent decompensations, these patients mostly present with mild HTG. This phenotype is often observed in heterozygous APOA5 loss of function mutations combined with various combinations of variants such as Haplotype Apo $A V^{*} 2$ and Apo $A V^{*} 3$ [33-35]. Between

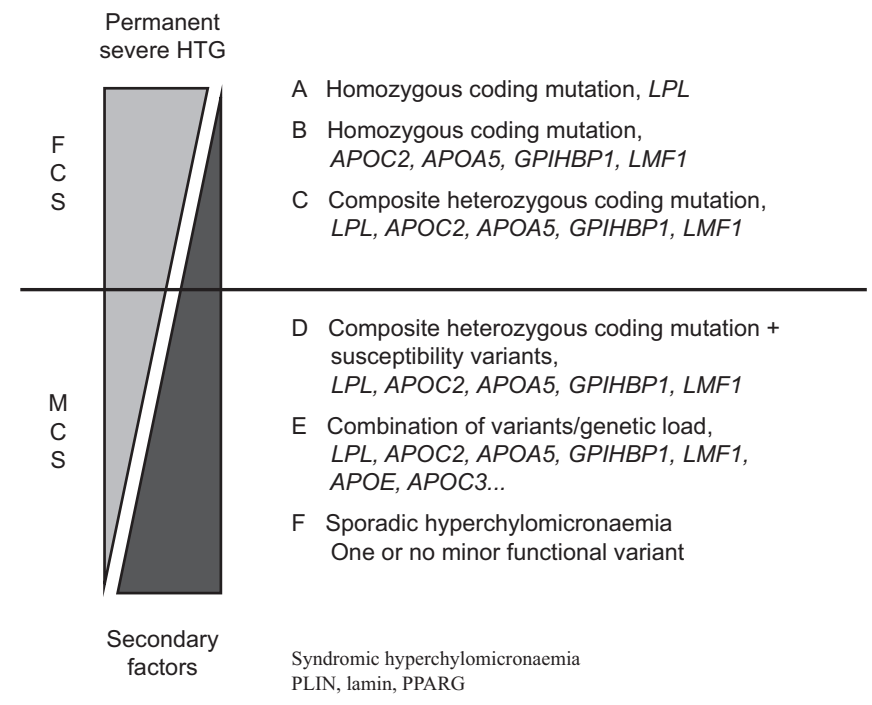

Fig. 2. Genetic load in FCS versus MCS.

FCS, familial chylomicronaemia syndrome; MCS, multifactorial chylomicronaemia syndrome; $L P L$, lipoprotein lipase; $A P O C 2$, apolipoprotein $C 2 ; A P O A 5$, apolipoprotein A5; GPIHBP1, glycosylphosphatidylinositol-anchored high-density lipoprotein-binding protein 1; LMF1, lipase maturation factor 1; $A P O E$, apolipoprotein E; APOC3, apolipoprotein C3; PPARG, peroxisome proliferator-activated receptor gamma.

decompensations, whereas plasma TG levels have partially decreased, post-heparin LPL activity is found to be normal $10 \mathrm{~min}$ after injection but is decreased in many patients 60 min after injection. This finding supports the hypothesis that some patients with MCS might have subtle alterations of LPL bioavailability [36]. Additionally, the apolipoprotein E2 allele is over-represented in patients with MCS; it is likely that the E2 allele contributes to decreases in hepatic chylomicron remnant clearance in patients carrying additional pathogenic variants known to alter LPL activity $[15,37]$. However, hyperchylomicronaemia is unusual even in E2/E2 patients, and they should be considered as suffering from MCS rather than FCS.

\subsection{Pivotal features needed for an appropriate diagnosis of FCS}

Differentiation of FCS from MCS is difficult, since the clinical and biological phenotype might be similar on decompensations. A number of parameters should be taken into consideration, given 
the fact that the post-heparin LPL activity assay we mentioned above is not commonly used in clinical practice, and that both FCS and MCS share mostly the same type V lipid phenotype. Therefore, TG levels over time, clinical signs and history must be all be considered for establishing a diagnosis of FCS. Although arbitrary, our proposed cut-off TG level for FCS diagnosis is fasting TG levels $>10 \mathrm{mmol} / \mathrm{L}$ or $885 \mathrm{mg} / \mathrm{dL}$. This is supported by a study of Italian patients with monogenic HTG, in which the lowest TG levels reported were $\sim 10 \mathrm{mmol} / \mathrm{L}$ [12].

It is advisable to check for a chylomicron layer by keeping plasma samples overnight when centrifugation is not able to provide evidence for a creamy layer. Screening for chylomicrons by agarose gel electrophoresis (lipidogram) is not reliable, since the largest chylomicrons often cannot enter the gel and consequently are not consistently detected. A TG/total cholesterol ratio $>5(\mathrm{mg} /$ $\mathrm{mg})>2.2(\mathrm{mmoL} / \mathrm{mmoL})$ and a plasma apolipoprotein B concentration $<100 \mathrm{mg} / \mathrm{dL}$ when plasma TG is $>10 \mathrm{mmol} / \mathrm{L}$ strongly support the presence of a high amount of chylomicrons and very large VLDL and remnants.

When the LPL activity assay can be performed ( 10 min after IV heparin injection [50 IU/kg]), LPL activity is found to be drastically decreased in homozygous LPL FCS and often lowered in cases of homozygous or compound heterozygous loss of function mutations in APOC2, APOA5, GPIHBP1 and LMF1 (Table 1). Reproducible increases of plasma TG $>10 \mathrm{mmol} / \mathrm{L}(885 \mathrm{mg} / \mathrm{dL})$ over several weeks or months is a relevant criterion supporting diagnosis of FCS. Plasma TG levels in MCS have more variability and are much more sensitive to dietary and/or fibrate treatment, whereas in FCS, TG concentration is minimally improved with these measures.

In addition to the lipid phenotype, some clinical features also support the diagnosis of FCS, rather than the more common MCS. FCS occurs in younger patients, mostly without secondary factors, except for pregnancy and contraceptive oral oestrogen use, whereas MCS typically occurs in overweight adult patients with metabolic syndrome. Moreover, the occurrence of AP is more frequent in FCS than in MCS due to the partial response to a low-fat diet and higher plasma TG concentrations [38,39].

Next-generation sequencing is likely to facilitate the diagnosis of FCS, since all the candidate genes can be tested in the same run. However, due to its cost and paucity in core laboratories, not all clinicians have access to genetic assessment.

We propose a pragmatic diagnostic score for FCS (Fig. 3), which might help to distinguish between FCS and MCS, based on eight biological/clinical items, including the history of plasma TG concentration, the lack of secondary factors (except for pregnancy and oestrogenic oral contraceptives), history of AP, no history of familial combined hyperlipidaemia [40] and age at the first onset of symptoms. A low weight was given to history of AP, since it is not specific to FCS versus MCS, although it seems to be more frequent in FCS. In addition, a large proportion of young patients with FCS do not yet have history of AP and would be excluded if the score gives too much weight to this item.

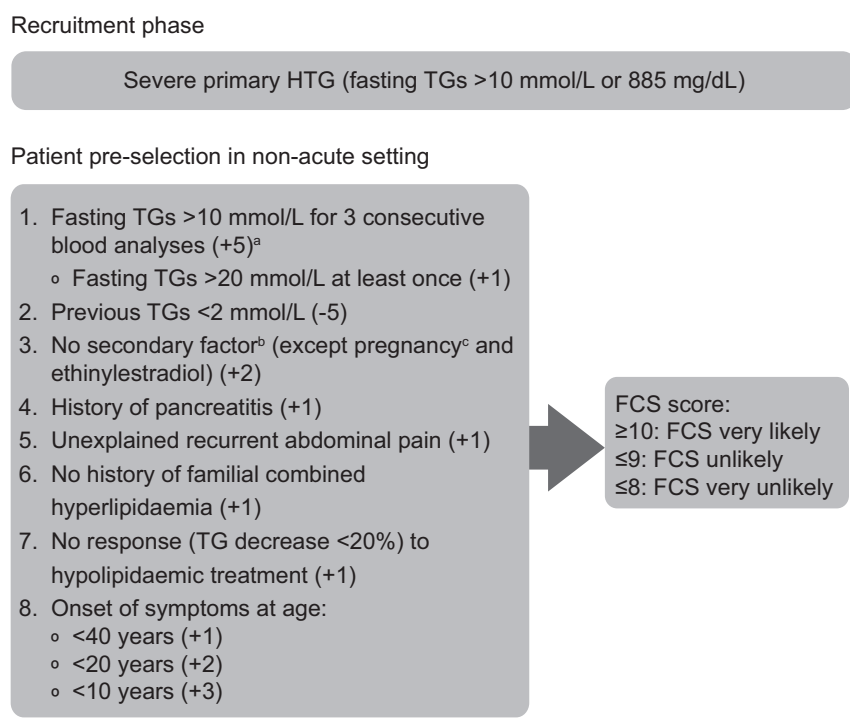

Fig. 3. A practical diagnostic scoring system for FCS.

Numbers in parentheses = weighting given to the presence of each item. FCS score $=$ the sum of all items present. FCS, familial chylomicronaemia syndrome; HTG, hypertriglyceridaemia; TG, triglyceride. ${ }^{\text {a }}$ Plasma TG concentration measured at least one month apart. Eruptive xanthoma may be used as a surrogate for high TG levels (rare). ${ }^{\text {b }}$ Secondary factors include alcohol, diabetes, metabolic syndrome, hypothyroidism, corticotherapy and additional drugs [6]. ${ }^{\mathrm{c}}$ If diagnosis is made during pregnancy, a second assessment is necessary to confirm diagnosis post-partum.

We initially tested the eight-item FCS score in two cohorts of 29 patients (see Methods in Ref. [41]). Sensitivity was estimated in an FCS cohort and specificity was tested in an MCS cohort (Figs. 3, 4A and B and Ref [41] Table 1).

The ability of the score to differentiate between FCS and MCS patients was subsequently tested in two independent replication Italian cohorts: the Rome Cohort (16 FCS and 15 MCS patients) and the Palermo Cohort (eight FCS and eight MCS patients) (Fig. 4C and D) [41]. The receiver operating characteristic (ROC) curve identified the best cut-off corresponding to an FCS score of $\geq 10$ (area under the ROC curve 0.91). Within the FCS replication cohorts, sensitivity (87.5\%) was similar in the Palermo and Rome cohorts; however, specificity tended to be lower in the Rome cohort than in the Palermo cohort ( $87.5 \%$ vs $66.6 \%$ ) (Fig. 5). Taking into account all the patients studied, the overall sensitivity was $88 \%$ (95\% confidence interval: $0.97,0.76$ ) with an overall specificity of $85 \%$ (95\% confidence interval: $0.94,0.75$ ).

\subsection{Discussion on diagnosis}

A clinical diagnosis scoring system seems to be the best approach to improve diagnosis of FCS. It would particularly facilitate the diagnosis of FCS in countries or areas where genetic testing is unavailable despite its decreasing costs.

Table 1

Phenotypes in FCS.

\begin{tabular}{|c|c|c|c|c|c|c|}
\hline & $L P L$ & APOC2 & APOA5 & GPIHBP1 & LMF1 & Anti-LPL/GPIHBP1 auto-antibodies ${ }^{\mathrm{a}}$ \\
\hline Transmission & Recessive & Recessive & Co-dominant? & Recessive & Recessive & Sporadic \\
\hline Variability of expressivity & + & ++ & ++++ & ++ & +++ & Hectic \\
\hline Initiation & Early & Early & Variable & Variable & Variable & Adult \\
\hline Therapeutic resistance & +++ & \pm & $+++1+$ & +++ & $?$ & +++ \\
\hline Relative frequency in FCS & $35-50 \%^{\mathrm{a}}$ & $5 \%$ & $10 \% ?$ & $5 \%$ & $1 \%$ & $<1 \%$ \\
\hline
\end{tabular}

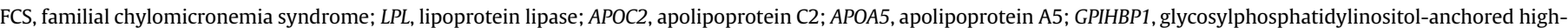
density lipoprotein-binding protein 1 ; LMF1, lipase maturation factor 1 .

${ }^{a}$ Relative frequency may be high in an area with a strong founder effect. 
A
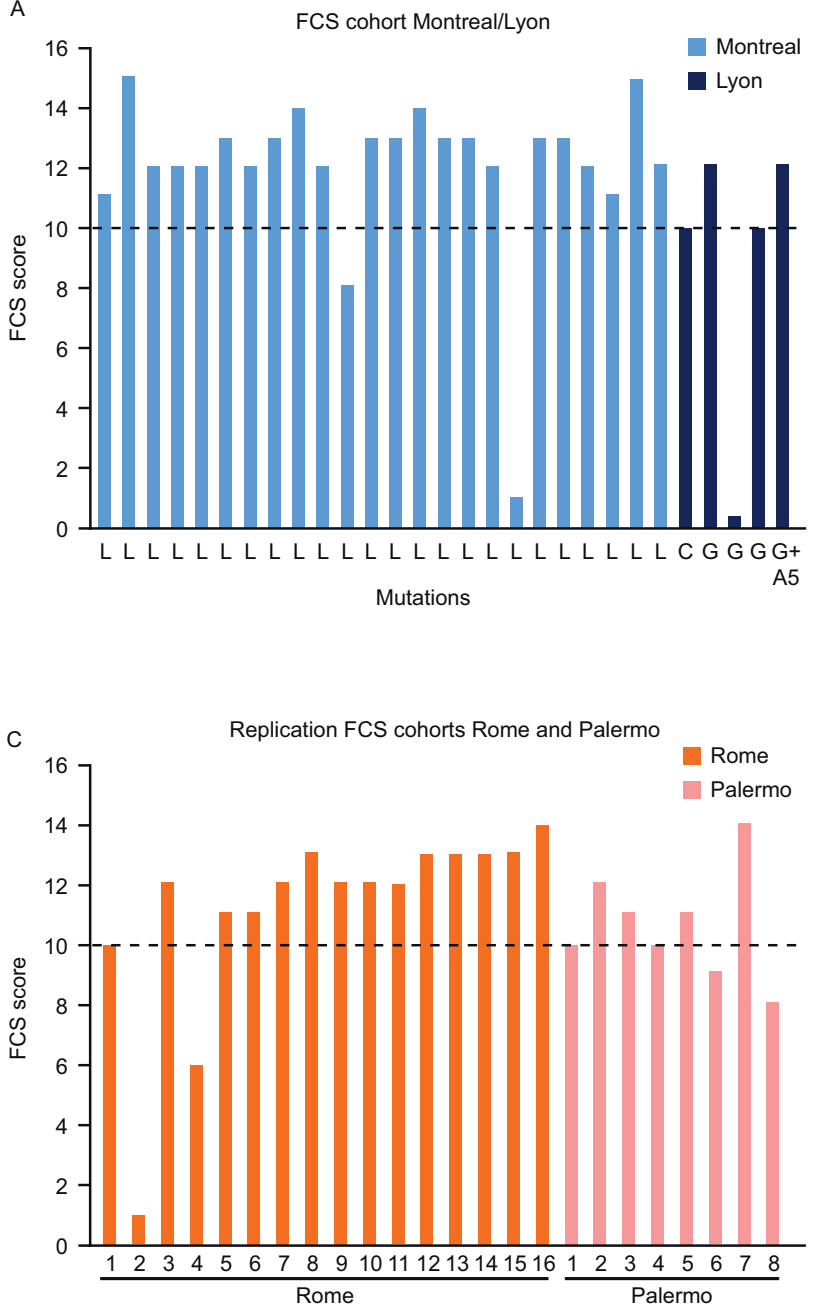

B
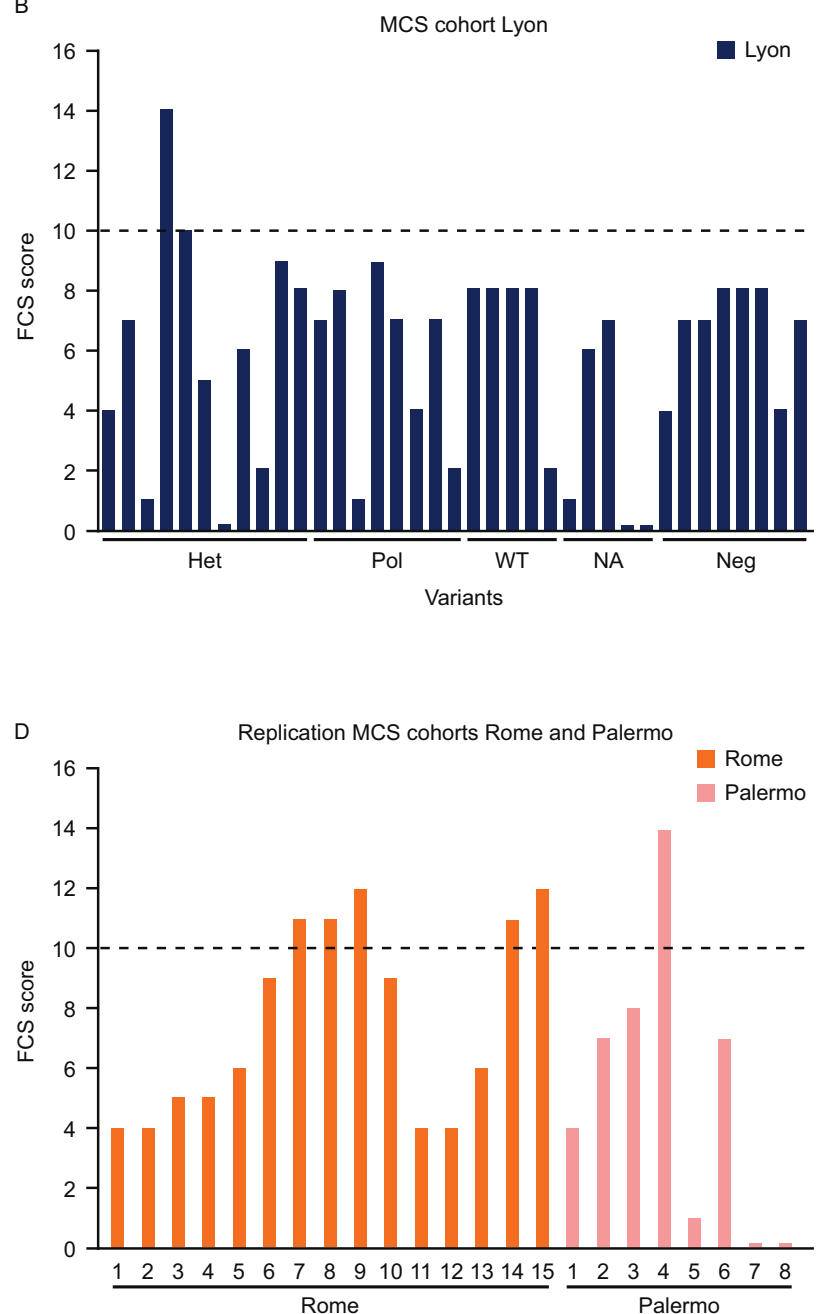

Fig. 4. Distribution of the FCS score in the different cohorts.

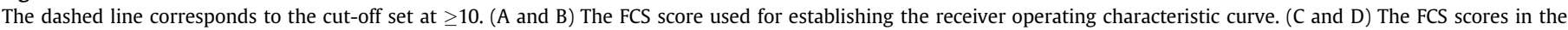

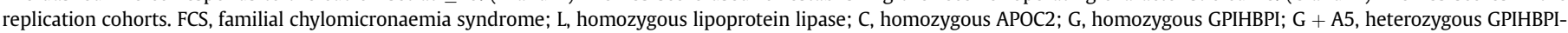
APOA5; Het, heterozygous coding mutation; Pol, multiple functional SNPs.

Although genetic data are the cornerstone of establishing the FCS diagnosis, it is sometimes difficult to assess the functionality of new variants. Conversely, in many patients with the FCS phenotype (and no evidence for auto-antibodies against LPL, APOC2 or GPIHBP1), a causative recessive loss of function mutation often remains elusive $[2,15]$. Pragmatic clinical scoring is likely to be a valuable phenotypic tool before performing next-generation sequencing is widely available. In patients with FCS, setting a lower cut-off than 10 would barely increase sensitivity in the two FCS cohorts, since only three out of 53 FCS patients had a very low score (FCS score 1 and 0 ), lower than most (83\%) of the patients with MCS (Fig. 4). This finding raises the question of unknown factors involved in the highly variable expressivity of the disease, such as additional genetic modulators.

The diagnostic performance of the score might be slightly lower in non-homozygous LPL FCS patients due to a milder phenotype. In addition, the sensitivity of the score might be mildly lower in European patients with FCS than in Canadian LPL-deficient patients. This may be due to the more mixed genotype, consequent to a lack of founder effect, and also to differences in nutrition and physical activity, leading to lower plasma TG concentration for similar low levels of LPL activity. This observation should be further tested in larger international registries. The general applicability of this scoring system in different geographical ancestries and ethnic backgrounds needs additional studies.

The lower specificity in the Rome cohort is likely due to the criteria used to enroll the Rome MCS cohort. In fact, these patients were selected for having hyperchylomicronaemia without any secondary factors; accordingly, most of them (73\%) were heterozygous for a coding mutation in one of the major genes involved in plasma TG regulation. Conversely, the MCS cohort in Lyon included all the consecutive MCS patients recruited for 2 years with a history of severe HTG, and most of them (90\%) had secondary factors. Consequently, it is likely that $66 \%$ might be the lowest specificity in severely hypertriglyceridaemic patients without any secondary factors and that in the usual clinical setting, specificity would be $>80 \%$, such as in Lyon and Palermo.

The setting of our pragmatic clinical score for this rare disease was based on an initial cohort of 29 patients with an established diagnosis of FCS, and the replication was tested in two smaller cohorts of FCS patients from Italy (24 patients); these numbers are rather large for such a rare disease. However, further validation in additional larger cohorts of patients with FCS from various countries with a different spectrum of loss of function mutations will be 


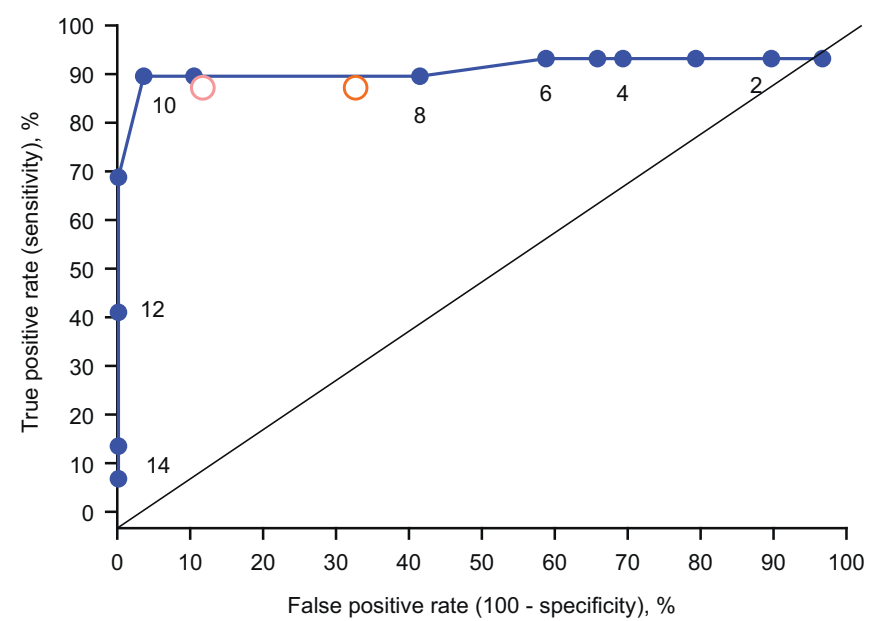

- Blue dots plotted according to sensitivity and specificity of the different levels of the FCS score

Cut-off $\geq 10$ Palermo Replication Cohort (8 FCS, 8 MCS)

Cut-off $\geq 10$ Rome Replication Cohort (16 FCS, 15 MCS)

Fig. 5. Sensitivity and specificity of the FCS score.

Receiver operating characteristic curve of the FCS score. FCS, familial chylomicronaemia syndrome; MCS, multifactorial chylomicronaemia syndrome.

needed. The FCS score might constitute a valuable tool for national and international registries, since it provides a way to take into account the differences in expressivity of the disease [22,42].

\subsection{Treatment goals}

The FCS score is a diagnostic tool. It should not be considered conditional for new-generation treatments. The indication of such treatments implies the assessment of the burden of the disease and especially the risk of recurrent hypertriglyceridaemic pancreatitis. The major aim of treatment for FCS is to reduce the risk of AP. Any management strategy should always aim to prevent pancreatitis events, which are characterised by marked increases in patient morbidity and mortality in FCS. Despite the fact that not all patients with HTG due to FCS develop pancreatitis, no biomarker is currently available to predict the risk of developing pancreatitis. Consequently, prevention of the occurrence of HTG remains the only valid target to avoid new episodes of pancreatitis.

TG levels $<10 \mathrm{mmol} / \mathrm{L}(<885 \mathrm{mg} / \mathrm{dL}$ ) are the most appropriate marker to reduce the risk of new episodes of AP. We suggest testing at least four times per year directly following pancreatitis in unstable patients and at least twice a year in stable patients without a history of HTG-induced pancreatitis. Blood withdrawal at the end of the afternoon [43] in the post-prandial state may be an option to challenge an apparently safe fasting plasma TG concentration. A strict hypolipidic diet remains the cornerstone of treatment but is often insufficient to avoid decompensation and recurrences of AP [2]. Since the majority of the TG-lowering interventions mediate their effect at least partially via LPL-mediated TG clearance, routine TG-lowering interventions are ineffective in FCS, including fibrate therapy and fish oil supplementation. Following the market withdrawal of the LPL gene therapy alipogene tiparvovec, current therapeutic options being investigated in FCS include apolipoprotein C3 antisense oligonucletotides (ASOs) [26,44] and angiopoietin-like 3 ASOs or monoclonal antibodies [45-47].

\section{Data gaps}

\subsection{Areas requiring further attention}

For patients with FCS, their symptoms, such as abdominal pain, fatigue and the ability to engage in daily activities, disease complications and adherence to a fat-restricted diet have an impact on their quality of life [48]. In a recent web-based survey of 60 US patients with FCS, a range of symptoms across the physical, emotional and cognitive domains were found to contribute to the burden of this illness [31]. More information is needed on how to reduce the handicap on social, work and family life due to abdominal pain, stress and strict diet restriction.

Since HTG has been found to correlate with decreased cognitive function in some but not all epidemiological studies [49,50], evaluating cognitive function in FCS with appropriate tools such as the Cambridge Neuropsychological Test Automated Battery could be considered [51].

Further investigation of the relationship between diet and pancreatitis is warranted. In addition to genetic factors, which need to be further identified [52-55], dietary factors related to pancreatitis, beyond those strictly related to TG levels such as alcohol, might be involved and could be a target. The mechanisms by which high levels of chylomicrons increase the risk of pancreatitis are not fully understood. Increased blood viscosity and impaired blood flow and peroxidative susceptibility causing pancreatic ischaemic and oxidative injury have been proposed as key factors [56,57]. Better understanding of these processes might create some translational opportunities.

\section{Conclusions}

Improvement of diagnosis of FCS is an important challenge in order to identify patients with the most severe form of HTG and with the highest risk of AP. Due to the strong similarities of the phenotype between FCS (considered as the recessive mono/bigenic disorder) and MCS (oligo/polygenic), the FCS score presented here provides a clinical tool able to discriminate between most cases. It offers the opportunity to focus genetic assessment in a targeted sub-population of hyperchylomicronaemic patients.

\section{Conflicts of interest}

PM has received fees paid to his institution for membership of advisory boards and clinical trials for Akcea/Ionis, Aegerion, Amgen, AMT/Chiesi, MSD, Novartis, Regeneron and Sanofi. RD has received honoraria for consulting from Amgen, Sanofi/Regeneron and Akcea/Ionis. MAv has received honoraria for membership of advisory boards and Speaker Bureaus for Akcea/Ionis, Aegerion, Amgen, MSD, Regeneron and Sanofi. MAr has received honoraria for membership of advisory boards and Speaker Bureaus for Akcea/ Ionis, Aegerion, Amgen, Regeneron and Sanofi. ABC has received honoraria for Speaker Bureaus for Alpha-Sigma, Aegerion, MSD and Sanofi. DN has received honoraria for lectures from MSD and Sanofi. LD'E has received grants from Aegerion/Amryt. ADC has nothing to declare. CM has nothing to declare. LAA-SW has received honoraria for Speaker Bureaus and lectures for Amgen, Regeneron/Sanofi, Sanofi-Aventis, MSD, Pfizer, Bayer, Ferrer, Esteve and Rovi, and consultancy fees for Akcea, MSD, Pfizer and Rovi. MB has received honoraria for Speaker Bureaus for Abbott/Mylan, Abbott Vascular, Actavis, Akcea, Amgen, Biofarm, KRKA, MSD, Sanofi-Aventis, Valeant and Zentiva, consultancy fees for Abbott Vascular, Akcea, Amgen, Daichii Sankyo, Esperion, Lilly, MSD, Resverlogix and Sanofi-Aventis, and grants from Sanofi and Valeant. JB has received research grants from Amgen, AstraZeneca, NovoNordisk, Pfizer and 
Regeneron/Sanofi, and honoraria for consultancy and lectures from Amgen, AstraZeneca, Eli Lilly, Merck, NovoNordisk, Pfizer and Regeneron/Sanofi. RC has presented data and chaired a meeting for Akcea, been on an advisory panel for Novartis, and is a trustee for HEART UK, The Cholesterol Charity. IG-B has received honoraria for consulting from Amgen, Sanofi, Eli Lilly, Regeneron, Akcea and Aegereon. EH reports personal fees from Akcea, outside the submitted work. CJ has received honoraria for consulting from Mylan, Abbott, UniQure and Akcea. XP has been involved in advisory boards with Amgen, Sanofi, Rubió, Ferrer and Esteve, has received honoraria from Akcea, and has given lectures for Mylan and Rubió. ZR has received honoraria for consulting from Akcea. JRvL has received honoraria from Akcea and grants from Aegerion/Amryt. HS reports personal fees from Akcea Therapeutics UK Ltd, during the conduct of the study, and grants and personal fees from Akcea Therapeutics UK Ltd, Amgen, Sanofi, Alexion Pharmaceuticals and Chiesi, outside the submitted work. CS has nothing to disclose. ES reports lecturing fees paid to his institute by Amgen, Sanofi, Chiesi, AstraZeneca, Regeneron and Akcea. EB declares having received honoraria for consulting/presentation from AstraZeneca, Amgen, Genfit, MSD, Sanofi, Regeneron, Unilever, Danone, Aegerion, Chiesi, Rottapharm-MEDA, Lilly, Ionis Pharmaceuticals and Akcea.

\section{Financial support}

The expert panel was initiated and supported by Akcea Therapeutics. The concepts addressed in this article were originally discussed at a meeting convened by Akcea for which participants, including some of the authors of this publication, received an honorarium. However, the authors received no payment in relation to the development of this publication, which was developed separately from the meeting. Akcea had no role and no support in the studies leading to establishment of the FCS score and did not interfere with the writing of the manuscript, except for funding editorial support.

\section{Author contributions}

The authors meet the criteria for authorship as recommended by the International Committee of Medical Journal Editors. They take full responsibility for the scope, direction, content of, and editorial decisions relating to the manuscript. The issues regarding FCS were discussed by PM, RD, MAv, MAr, LAS, MB, JB, RC, IGB, EH, CJ, XP, ZR, JRVL, HS, CS, ES and EB. The data for establishing the FCS score were generated and collected by RD, MAr, MAv, ABC, DN, LDE, $\mathrm{ADC}, \mathrm{CM}$ and PM. The discussion regarding the topic was contributed to by RD, MAr, MAv, PM, ES and EB. The data were analysed by $\mathrm{RD}, \mathrm{MAr}, \mathrm{MAv}, \mathrm{PM}$ and EB. The manuscript was written by PM and EB. All authors reviewed the manuscript.

\section{Acknowledgements}

Medical writing assistance was provided by Karen Brayshaw, $\mathrm{PhD}$, of Complete HealthVizion, which was contracted and compensated by Akcea Therapeutics.

We thank Marine Ginoux from Pharmaco Epidémiologie Lyon for providing assistance in the establishment of the ROC curve.

\section{References}

[1] J.R. Burnett, A.J. Hooper, R.A. Hegele, Familial lipoprotein lipase deficiency https://www.ncbi.nlm.nih.gov/books/NBK1308/, 2017. (Accessed 15 February 2018).

[2] A.J. Brahm, R.A. Hegele, Chylomicronaemia-current diagnosis and future therapies, Nat. Rev. Endocrinol. 11 (6) (2015) 352-362.

[3] T. Normand, J. Bergeron, T. Fernandez-Margallo, A. Bharucha, M.R. Ven
Murthy, P. Julien, et al., Geographic distribution and genealogy of mutation 207 of the lipoprotein lipase gene in the French Canadian population of Québec, Hum. Genet. 89 (6) (1992) 671-675.

[4] M.F. Linton, A.H. Hasty, V.R. Babaev, S. Fazio, Hepatic apo E expression is required for remnant lipoprotein clearance in the absence of the low density lipoprotein receptor, J. Clin. Invest. 101 (8) (1998) 1726-1736.

[5] A.P. Beigneux, K. Miyashita, M. Ploug, D.J. Blom, M. Ai, M.R.F. Linton, et al., Autoantibodies against GPIHBP1 as a cause of hypertriglyceridemia, N. Engl. J. Med. 376 (17) (2017) 1647-1658.

[6] A.L. Catapano, I. Graham, G. De Backer, O. Wiklund, M.J. Chapman, H. Drexel, et al., 2016 ESC/EAS Guidelines for the management of dyslipidaemias. The task force for the management of dyslipidaemias of the European society of cardiology (ESC) and European atherosclerosis society (EAS) developed with the special contribution of the European association for cardiovascular prevention \& rehabilitation (EACPR), Atherosclerosis 253 (2016) 281-344.

[7] C. Dionisi-Vici, E. Shteyer, M. Niceta, C. Rizzo, B. Pode-Shakked, G. Chillemi, et al., Expanding the molecular diversity and phenotypic spectrum of glycerol 3phosphate dehydrogenase 1 deficiency, J. Inherit. Metab. Dis. 39 (5) (2016) 689-695.

[8] R.A. Hegele, H.N. Ginsberg, M.J. Chapman, B.G. Nordestgaard, J.A. Kuivenhoven, M. Averna, et al., The polygenic nature of hypertriglyceridaemia: implications for definition, diagnosis, and management, Lancet Diabetes Endocrinol 2 (8) (2014) 655-666.

[9] C.T. Johansen, R.A. Hegele, Genetic bases of hypertriglyceridemic phenotypes, Curr. Opin. Lipidol. 22 (4) (2011) 247-253.

[10] M. Joshi, J. Eagan, N.K. Desai, S.A. Newton, M.C. Towne, N.S. Marinakis, et al., A compound heterozygous mutation in GPD1 causes hepatomegaly, steatohepatitis, and hypertriglyceridemia, Eur. J. Hum. Genet. 22 (10) (2014) 1229-1232.

[11] J.J. Rios, S. Shastry, J. Jasso, N. Hauser, A. Garg, A. Bensadoun, et al., Deletion of GPIHBP1 causing severe chylomicronemia, J. Inherit. Metab. Dis. 35 (3) (2012) $531-540$.

[12] C. Rabacchi, L. Pisciotta, A.B. Cefalù, D. Noto, R. Fresa, P. Tarugi, et al., Spectrum of mutations of the LPL gene identified in Italy in patients with severe hypertriglyceridemia, Atherosclerosis 241 (1) (2015) 79-86.

[13] R.P. Surendran, M.E. Visser, S. Heemelaar, J. Wang, J. Peter, J.C. Defesche, et al., Mutations in LPL, APOC2, APOA5, GPIHBP1 and LMF1 in patients with severe hypertriglyceridaemia, J. Intern. Med. 272 (2) (2012) 185-196.

[14] R.A. Carr, B.J. Rejowski, G.A. Cote, H.A. Pitt, N.J. Zyromski, Systematic review of hypertriglyceridemia-induced acute pancreatitis: a more virulent etiology? Pancreatology 16 (4) (2016) 469-476.

[15] W.V. Brown, D. Gaudet, I. Goldberg, R. Hegele, Roundtable on etiology of familial chylomicronemia syndrome, J. Clin. Lipidol. 12 (1) (2018) 5-11.

[16] Z. Ahmad, R. Halter, M. Stevenson, Building a better understanding of the burden of disease in familial chylomicronemia syndrome, Expet Rev. Clin. Pharmacol. 10 (1) (2017) 1-3.

[17] D. Gaudet, J. de Wal, K. Tremblay, S. Déry, S. van Deventer, A. Freidig, et al., Review of the clinical development of alipogene tiparvovec gene therapy for lipoprotein lipase deficiency, Atherosclerosis Suppl. 11 (1) (2010) 55-60.

[18] J.D. Brunzell, A. Chait, E.L. Bierman, Pathophysiology of lipoprotein transport, Metabolism 27 (9) (1978) 1109-1127.

[19] R.A. Hegele, M.R. Ban, N. Hsueh, B.A. Kennedy, H. Cao, G.Y. Zou, et al., A polygenic basis for four classical Fredrickson hyperlipoproteinemia phenotypes that are characterized by hypertriglyceridemia, Hum. Mol. Genet. 18 (21) (2009) 4189-4194.

[20] R.M. Krauss, R.I. Levy, D.S. Fredrickson, Selective measurement of two lipase activities in postheparin plasma from normal subjects and patients with hyperlipoproteinemia, J. Clin. Invest. 54 (5) (1974) 1107-1124.

[21] V. Pruneta, P. Moulin, F. Labrousse, P.J. Bondon, G. Ponsin, F. Berthezene, Characterization of a new case of autoimmune type I hyperlipidemia: longterm remission under immunosuppressive therapy, J. Clin. Endocrinol. Metab. 82 (3) (1997) 791-796.

[22] E. Steinhagen-Thiessen, E. Stroes, H. Soran, C. Johnson, P. Moulin, G. Iotti, et al., The role of registries in rare genetic lipid disorders: review and introduction of the first global registry in lipoprotein lipase deficiency, Atherosclerosis 262 (2017) 146-153.

[23] E. Stroes, P. Moulin, K.G. Parhofer, V. Rebours, J.-M. Löhr, M. Averna, Diagnostic algorithm for familial chylomicronemia syndrome, Atherosclerosis Suppl. 23 (2017) 1-7.

[24] C. Stefanutti, G. Labbadia, C. Morozzi, Severe hypertriglyceridemia-related acute pancreatitis, Ther. Apher. Dial. 17 (2) (2013) 130-137.

[25] L.-H. Deng, P. Xue, Q. Xia, X.-N. Yang, M.-H. Wan, Effect of admission hypertriglyceridemia on the episodes of severe acute pancreatitis, World J. Gastroenterol. 14 (28) (2008) 4558-4561.

[26] D. Gaudet, D. Brisson, K. Tremblay, V.J. Alexander, W. Singleton, S.G. Hughes, et al., Targeting APOC3 in the familial chylomicronemia syndrome, N. Engl. J. Med. 371 (23) (2014) 2200-2206.

[27] G. Sisman, Y. Erzin, I. Hatemi, E. Caglar, S. Boga, V. Singh, et al., Familial chylomicronemia syndrome related chronic pancreatitis: a single-center study, Hepatobiliary Pancreat. Dis. Int. 13 (2) (2014) 209-214.

[28] M.J. Murphy, X. Sheng, T.M. MacDonald, L. Wei, Hypertriglyceridemia and acute pancreatitis, JAMA Intern, Med 173 (2) (2013) 162-164.

[29] G. Yuan, K.Z. Al-Shali, R.A. Hegele, Hypertriglyceridemia: its etiology, effects and treatment, CMAJ (Can. Med. Assoc. J.) 176 (8) (2007) 1113-1120.

[30] S.B. Pedersen, A. Langsted, B.G. Nordestgaard, Nonfasting mild-to-moderate 
hypertriglyceridemia and risk of acute pancreatitis, JAMA Intern. Med 176 (12) (2016) 1834-1842.

[31] M. Davidson, M. Stevenson, A. Hsieh, Z. Ahmad, C. Crowson, J.L. Witztum, The burden of familial chylomicronemia syndrome: interim results from the INFOCUS study, Expert Rev. Cardiovasc Ther. 15 (5) (2017) 415-423.

[32] A. Gelrud, K.R. Williams, A. Hsieh, A.R. Gwosdow, A. Gilstrap, A. Brown, The burden of familial chylomicronemia syndrome from the patients' perspective, Expert Rev. Cardiovasc Ther. 15 (11) (2017) 879-887.

[33] P.J. Talmud, E. Hawe, S. Martin, M. Olivier, G.J. Miller, E.M. Rubin, et al. Relative contribution of variation within the APOC3/A4/A5 gene cluster in determining plasma triglycerides, Hum. Mol. Genet. 11 (24) (2002) 3039-3046.

[34] C. Caussy, S. Charrière, C. Marçais, M. Di Filippo, A. Sassolas, M. Delay, et al., An APOA5 3' UTR variant associated with plasma triglycerides triggers APOA5 downregulation by creating a functional miR-485-5p binding site, Am. J. Hum. Genet. 94 (1) (2014) 129-134.

[35] C. Marçais, B. Verges, S. Charrière, V. Pruneta, M. Merlin, S. Billon, et al., Apoa5 Q139X truncation predisposes to late-onset hyperchylomicronemia due to lipoprotein lipase impairment, J. Clin. Invest. 115 (10) (2005) 2862-2869.

[36] O. Marmontel, M. Di Filippo, C. Marcais, S. Nony, M. Dumoux, M. ServeauxDancer, et al., Alterations in plasma triglycerides lipolysis in patients with history of multifactorial chylomicronemia, Atherosclerosis 265 (2017) 22-28.

[37] S. Charrière, C. Cugnet, M. Guitard, S. Bernard, L. Groisne, M. Charcosset, et al. Modulation of phenotypic expression of APOA5 Q97X and L242P mutations, Atherosclerosis 207 (1) (2009) 150-156.

[38] J.D. Brunzell, S.S. Deeb, Familial lipoprotein lipase deficiency, Apo C-II deficiency, and hepatic lipase deficiency, in: C.R. Scriver, A.L. Beaudet, D. Valle, et al. (Eds.), The Metabolic and Molecular Bases of Inherited Disease, McGrawHill, 2001, pp. 2789-2816.

[39] T. Gotoda, K. Shirai, T. Ohta, J. Kobayashi, S. Yokoyama, S. Oikawa, et al. Diagnosis and management of type I and type $\mathrm{V}$ hyperlipoproteinemia, J. Atherosclerosis Thromb. 19 (1) (2012) 1-12.

[40] M.J. Veerkamp, J. de Graaf, S.J.H. Bredie, J.C.M. Hendriks, P.N.M. Demacker, A.F.H. Stalenhoef, Diagnosis of familial combined hyperlipidemia based on lipid phenotype expression in 32 families. Results of a 5-year follow-up study, Arterioscler. Thromb. Vasc. Biol. 22 (2) (2002) 274-282.

[41] P. Moulin, R. Dufour, M. Averna, M. Arca, A.B. Cefalù, D. Noto, et al., Data on Characterisation of Patients with Familial Chylomicronaemia Syndrome (FCS) and Multifactorial Chylomicronaemia Syndrome (MCS) Included in the Establishment of the FCS Clinical Diagnostic Score, 2018. Atherosclerosis Data in Brief Submitted.

[42] D.M. Ng, A.J. Hooper, M.I. Bellgard, J.R. Burnett, The role of patient registries for rare genetic lipid disorders, Curr. Opin. Lipidol. 29 (2) (2018) 156-162.

[43] M. Castro Cabezas, C.J.M. Halkes, S. Meijssen, A.J.H.H.M. van Oostrom, D.W. Erkelens, Diurnal triglyceride profiles: a novel approach to study triglyceride changes, Atherosclerosis 155 (1) (2001) 219-228.

[44] D. Gaudet, V. Alexander, B.F. Baker, D. Brisson, K. Tremblay, W. Singleton, et al., Antisense inhibition of apolipoprotein C-III in patients with hypertriglyceridemia, N. Engl. J. Med. 373 (5) (2015) 438-447.

[45] D. Gaudet, Novel therapies for severe dyslipidemia originating from human genetics, Curr. Opin. Lipidol. 27 (2) (2016) 112-124.

[46] ClinicalTrials.Gov, NCT0336074: Phase 2 study of AKCEA-ANGPTL3-LRX (ISIS 703802) in patients with familial chylomicronemia syndrome (FCS). https:/ clinicaltrials.gov/ct2/show/NCT03360747?term=angPTL\&rank=2, 2017. (Accessed 3 February 2018)

[47] ClinicalTrials, Gov, NCT03371355: study of ISIS 703802 in subjects with hypertriglyceridemia, type 2 diabetes Mellitus, and Nonalcoholic fatty liver disease. https://clinicaltrials.gov/ct2/show/NCT03371355? term=angPTL\&rank=4, 2017. (Accessed 3 February 2018).

[48] C. Johnson, E.S. Stroes, H. Soran, A. Wierzbicki, P. Moulin, E. Bruckert, et al. Issues affecting quality of life and disease burden in lipoprotein lipase deficiency (LPLD) - first step towards a PRO measure in LPLD, Value Health 18 (7) (2015) A707 (Abstract no. PRM141).

[49] C.-Q. Huang, B.-R. Dong, H.-M. Wu, Y.-L. Zhang, J.-H. Wu, Z.-C. Lu, et al., Association of cognitive impairment with serum lipid/lipoprotein among Chinese nonagenarians and centenarians, Dement. Geriatr. Cognit. Disord. 27 (2) (2009) 111-116.

[50] J.E. Morley, W.A. Banks, Lipids and cognition, J. Alzheimers Dis 20 (3) (2010) 737-747.

[51] T.W. Robbins, M. James, A.M. Owen, B.J. Sahakian, L. McInnes, P. Rabbitt Cambridge Neuropsychological Test Automated Battery (CANTAB): a facto analytic study of a large sample of normal elderly volunteers, Dementia 5 (5) (1994) 266-281.

[52] B. Hölzl, H.G. Kraft, H. Wiebusch, A. Sandhofer, J. Patsch, F. Sandhofer, et al., Two novel mutations in the lipoprotein lipase gene in a family with marked hypertriglyceridemia in heterozygous carriers: potential interaction with the polymorphic marker D1S104 on chromosome 1q21-q23, J. Lipid Res. 41 (5) (2000) 734-741.

[53] I. Lamiquiz-Moneo, C. Blanco-Torrecilla, A.M. Bea, R. Mateo-Gallego, S. PérezCalahorra, L. Baila-Rueda, et al., Frequency of rare mutations and common genetic variations in severe hypertriglyceridemia in the general population of Spain, Lipids Health Dis. 15 (2016) 82

[54] G.F. Lewis, C. Xiao, R.A. Hegele, Hypertriglyceridemia in the genomic era: a new paradigm, Endocr. Rev. 36 (1) (2015) 131-147.

[55] J. Scherer, V.P. Singh, C.S. Pitchumoni, D. Yadav, Issues in hypertriglyceridemic pancreatitis: an update, J. Clin. Gastroenterol. 48 (3) (2014) 195-203.

[56] A.S. Khokhar, D.L. Seidner, The pathophysiology of pancreatitis, Nutr. Clin. Pract. 19 (1) (2004) 5-15.

[57] P. Valdivielso, A. Ramírez-Bueno, N. Ewald, Current knowledge of hypertriglyceridemic pancreatitis, Eur. J. Intern. Med. 25 (8) (2014) 689-694. 